\title{
Screening Tools as a Predictor of Injury in Gymnastics: Systematic Literature Review
}

\author{
Ross Armstrong ${ }^{1^{*}}$ (D) and Nicola Relph² (D)
}

\begin{abstract}
Background: Gymnastics requires a high level of physical ability and technical skill which utilises short sets of athleticism and artistry to perform complex and intense movements which can overload musculoskeletal tissues and result in acute injuries which can develop into chronic injuries. The aim of this systematic literature review was to investigate which screening tools predict injury in gymnasts and encompasses all genres, levels and ages.

Methods: An electronic search of seven databases from their inception until March 2021 was conducted. The databases were the Allied and Complementary Medicine Database, CINAHL, eBook Collection (EBSCOhost), MEDLINE, Cochrane Database of Systematic Reviews, SPORTDiscus and PEDro (the Physiotherapy Evidence Base). A combination of the following search terms was used: (1) Gymnastics AND injury AND Screening, (2) Screening AND Gymnastics and (3) Musculoskeletal AND Screening AND Gymnastics. These terms were searched in all text, abstract, title and subject terms. Studies were assessed using a 20-point scoring tool.
\end{abstract}

Results: The mean methodological quality score was 13.1 points (range 10-17 points). Range of motion, anthropometric and postural measurements, hypermobility, clinical diagnostic tests, movement screening tools, muscle strength, power and endurance were reported in the included studies. Some evidence existed for screening measurement of height and mass as taller and heavier gymnasts might be more susceptible to injury; however, the different methodologies utilised and lack of acknowledgment of confounding variables limit the clinical relevance of these findings.

Conclusions: Height and mass should be recorded during the screening process. A lack of heterogeneity in study methodology prevented a meta-analysis. Studies were limited by a lack of prospective injury design, poor injury definition, self-reporting of injury and only 2 studies reported reliability of screening tools. Further research is required to determine the role of injury screening in gymnastics.

Registration: The review protocol was registered with the International Prospective Register of Systematic Reviews (PROSPERO) with the registration number CRD42020218339.

Keywords: Gymnastics, Height, Weight, Body mass index, Injury, Pain

\section{Key Points}

- Taller and heavier gymnasts might be moresusceptible to injury.
- Studies have investigated a range of screeningmeasurements.

- There is a need for prospective studies that defineinjury and measure the reliability of screening tools used.

\footnotetext{
*Correspondence: ross.armstrong@cumbria.ac.uk

${ }^{1}$ Rehabilitation and Healthy Lives Research Group, Institute of Health,

University of Cumbria, Carlisle, Cumbria CA1 2HH, England

Full list of author information is available at the end of the article
} 


\section{Background}

Gymnastics requires a high level of physical ability and technical skill [1] which utilises short sets of athleticism and artistry to perform complex and intense movements. These athletic characteristics include strength, speed, power, agility, cardiovascular endurance, flexibility, coordination and balance. Gymnastics can involve several disciplines including rhythmic, artistic, trampoline, acrobatic and aerobic gymnastics all of which have different physical demands. Gymnastics requires repetitive movements which can overload joints and result in acute injuries which can develop into chronic injuries. These loads combined with movements that regularly exceed normal anatomical range can potentially result in injury. In aerobic and acrobatic gymnastics, the high volume of throws and catches in the jump elements creates high impact loading in lower extremity joints [2]. As the demands and rewards of competition increase it is likely that gymnasts will continue to work on the periphery of a sustainable training load and without sufficient rest the injury risk will remain high.

Injury rates of $1.08 / 1000 \mathrm{~h}$ participation have been reported in rhythmic gymnastics [3]. Injury rates per $1000 \mathrm{~h}$ for artistic gymnastics participation range from 0.427 [4] in lower-level female gymnasts to 22.7 [5] in intercollegiate competition gymnasts. Within artistic gymnastics the floor apparatus is associated with the greatest injury risk [6-8]. Gymnastic movements rely heavily on integration via the kinetic chain with skills such as handsprings and walkovers involving spine and hip extension and shoulder hyperflexion [9]. Injury surveillance of 3 Olympic games has identified that the lower limb (62.8\%), trunk (23.1\%) and upper limb (14.1\%) as the regions most susceptible to injury with the ankle the most prominent location (21.8\%) [10]. The dominance of lower limb injury is supported across competitive levels in further studies $[6,7,11]$. Therefore, there is a high risk of injury in gymnastics regardless of discipline and level. Injury can have a detrimental impact on a gymnast's health and well-being, and therefore, injury prevention interventions including screening are essential.

The Van Mechelen model of injury prevention [12] and the development of injury prevention programmes requires injury surveillance to identify best practice and potential interventions. One such intervention is the use of screening tools to identify athletes that are at risk of injury [13-16]. The determination and implementation of effective screening tools may have a positive physical and psychological impact on gymnasts by potentially reducing injury risk via the implementation of injury prevention programmes. The current systematic literature review is the first to investigate which screening tools can predict injury in gymnasts and encompasses all genres, levels and ages. A meta-analysis was also proposed to synthesise similar data sets where appropriate.

\section{Methods}

The review protocol was registered with the International Prospective Register of Systematic Reviews (PROSPERO) [17] with the registration number CRD42020218339.

\section{Search Strategy}

A systematic literature search was conducted to obtain articles regarding screening tools that can potentially predict injury in gymnasts from the inception of seven databases until March 2021. The databases were the Allied and Complementary Medicine Database (AMED), CINAHL, eBook Collection (EBSCOhost), MEDLINE, Cochrane Database of Systematic Reviews, SPORTDiscus and PEDro (the Physiotherapy Evidence Base). A combination of the following search terms was used: (1) Gymnastics AND injury AND Screening, (2) Screening AND Gymnastics and (3) Musculoskeletal AND Screening AND Gymnastics. These terms were searched in all text, abstract, title and subject terms. Reference lists of acquired articles were screened to find additional articles, and duplicates were removed.

\section{Study Selection}

The titles and abstracts of the search returned articles were reviewed by the first author (RA) to identify potential relevance using a two-stage process. The first stage involved the classification of articles as relevant, potentially relevant or irrelevant. During this stage, irrelevant articles were excluded, and articles that met the inclusion criteria were retained for further analysis. The second stage involved the review of the full text of relevant and potentially relevant articles by two reviewers (RA and NR). Both reviewers formulated comments regarding the suitability of articles using the checklist of five inclusion criteria and then met to determine final inclusion via reviewing these comments. Any potential disagreements regarding the inclusion were referred to a third reviewer to determine final inclusion. Studies were included if they were (1) full text, (2) in the English language, (3) used a screening tool and/or physical measurement, (4) the population was gymnasts and (5) injury or pain occurrence was reported either retrospectively or prospectively. This review only included screening tools that can be utilised in the field. Therefore, studies that utilised equipment such as thermal imaging, force plates and computerised dynamic posturography were excluded as they were deemed to be laboratory-based and limited in the practical application of gymnastic injury screening. 


\section{Data Extraction}

Two reviewers (RA and NR) independently extracted data from each article. The following information was extracted if available: study design (prospective or retrospective), level of evidence, location of testing, inclusion and exclusion criteria, subject characteristics (age, sex, height, weight); screening tool and/or physical measurements recorded; reliability and validity of screening tool and/or physical measurements and method of injury collection including retrospective/prospective injury collection, definition of injury, individual diagnosing injury, statistical analysis of injury measure, percentage of missing data or withdrawals, outcome measures and identification of confounders.

\section{Methodological Quality}

A previous review of injury screening tools in dance [18] utilised a 20-point scoring system and the authors provided permission for use of this tool in the current review. This scoring tool was developed from a previously published screening tool in team sports [19] and modified version Cochrane Group on Screening and Diagnostic Test Methodology (Cochrane methods) [20]. The scoring system is outlined in Table 1. For study design, those studies that included both retrospective and prospective injury data collection were awarded 1 point. The level of evidence devised from the Oxford Centre for EvidenceBased Medicine ranged from 1 to 5 , with 1 the lowest and 5 the highest score. Both inclusion and exclusion criteria had to be stated to score 1 point and setting information needed to include the name of the venue for $1 \mathrm{pt}$. Demographic information needed to contain a minimum of age and gender to score $1 \mathrm{pt}$. The screening tool needed to be described sufficiently to allow replication to score $1 \mathrm{pt}$. An effective injury definition and diagnosis by an appropriate professional (e.g. physiotherapist and/or doctor) is essential [18]; therefore, the presence of both criteria was awarded 1pt each. The methodological score based on statistical analysis was divided into two separate questions. The study was awarded 1 point if it had included an inferential statistical analysis of any kind. However, the study was awarded an additional point if a regression model or risk measurement had been applied; in the current review, this included linear regression models, logistical regression models, Cox regression models, odds ratio (OR) analysis and relative risk (RR) analysis. This aspect of the methodological quality score allowed differentiation between studies that consider the injury screening tool predictive capability and those that did not. The studies which considered only the ability of the screening tool to identify the differences between the injured and non-injured groups were not awarded this additional point. The reliability of tools is a fundamental component of an effective methodology, and therefore, 1 point was awarded for studies that reported reliability from previous studies and 2 points for those that reported the reliability from the actual study data. Those studies that reported the withdrawal of participants and provided information regarding missing data were awarded $1 \mathrm{pt}$. For outcome measures studies were awarded 1 point if the outcome measures were clearly reported and studies were awarded 1 point if the confounders were reported.

\section{Data Analysis}

A meta-analysis was considered for all outcome measures. Four studies reported body weight for injured and non-injured gymnasts [4, 9, 23, 25]. However, the age of gymnasts in these studies ranged from 5 to 19 years and hence it was not appropriate to pool data. Age differences in the samples were also the reason for not pooling height data provided by 3 studies $[4,9,25]$ and waist circumference data from two studies [4, 23]. Grip strength of gymnasts was measured by [4, 25]; however, different data analysis procedures were used, only one study normalised the scores by body weight [4], and hence again, pooling was not possible. Finally, vertical jump [1, 4, 29] and quadriceps angle ( $\mathrm{Q}$ angle) data $[2,24]$ were considered for meta-analysis; however, inconsistency in protocols and data presentation prevented pooling of the data.

\section{Results}

\section{Included Studies}

The initial search yielded 8376 studies for review. The title and abstracts of these articles were reviewed, and duplicates removed, which resulted in 6263 articles requiring further consideration. Assessment of the eligibility of the full text of these articles and the application of inclusion and exclusion criteria meant that 15 articles were included in the systematic review. Figure 1 outlines the search strategy [32]. The assessment of the methodological quality is reported in Table 1 . The mean score was 13.1 points (range $10-17$ points). Table 2 reports the characteristics of these studies.

\section{Gymnastic Genre and Level}

Two studies included artistic gymnasts [21, 22], 2 studies included rhythmic gymnasts [23, 24], 11 studies simply used the term "gymnasts" [1, 2, 4, 9, 25-31]. With regard to the level of gymnasts, 6 studies included gymnasts classified as national team level [9, 21, 22, 29-31], 2 studies involved gymnasts from clubs affiliated with a national federation [1,23], 1 study involved gymnasts 


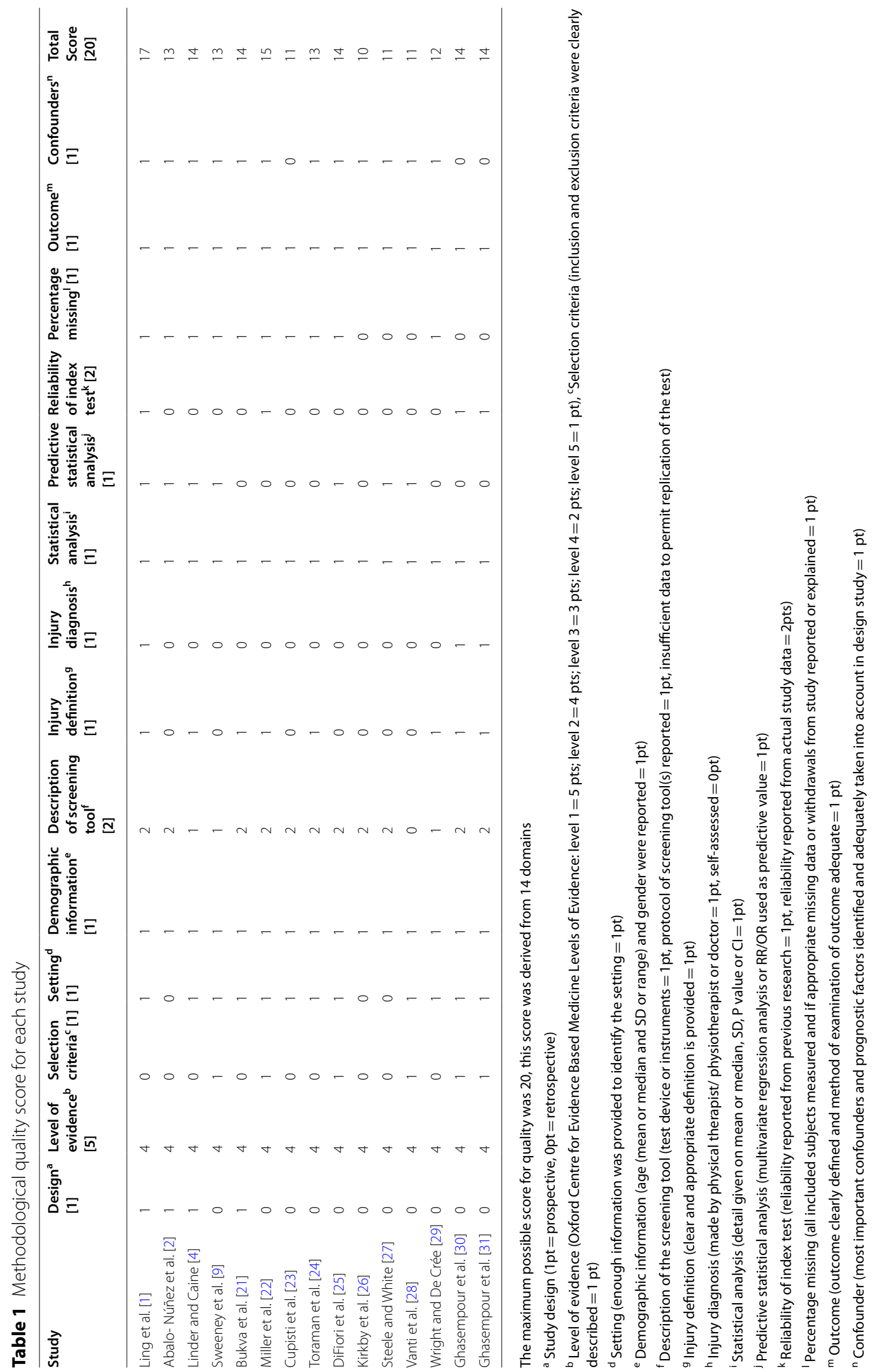




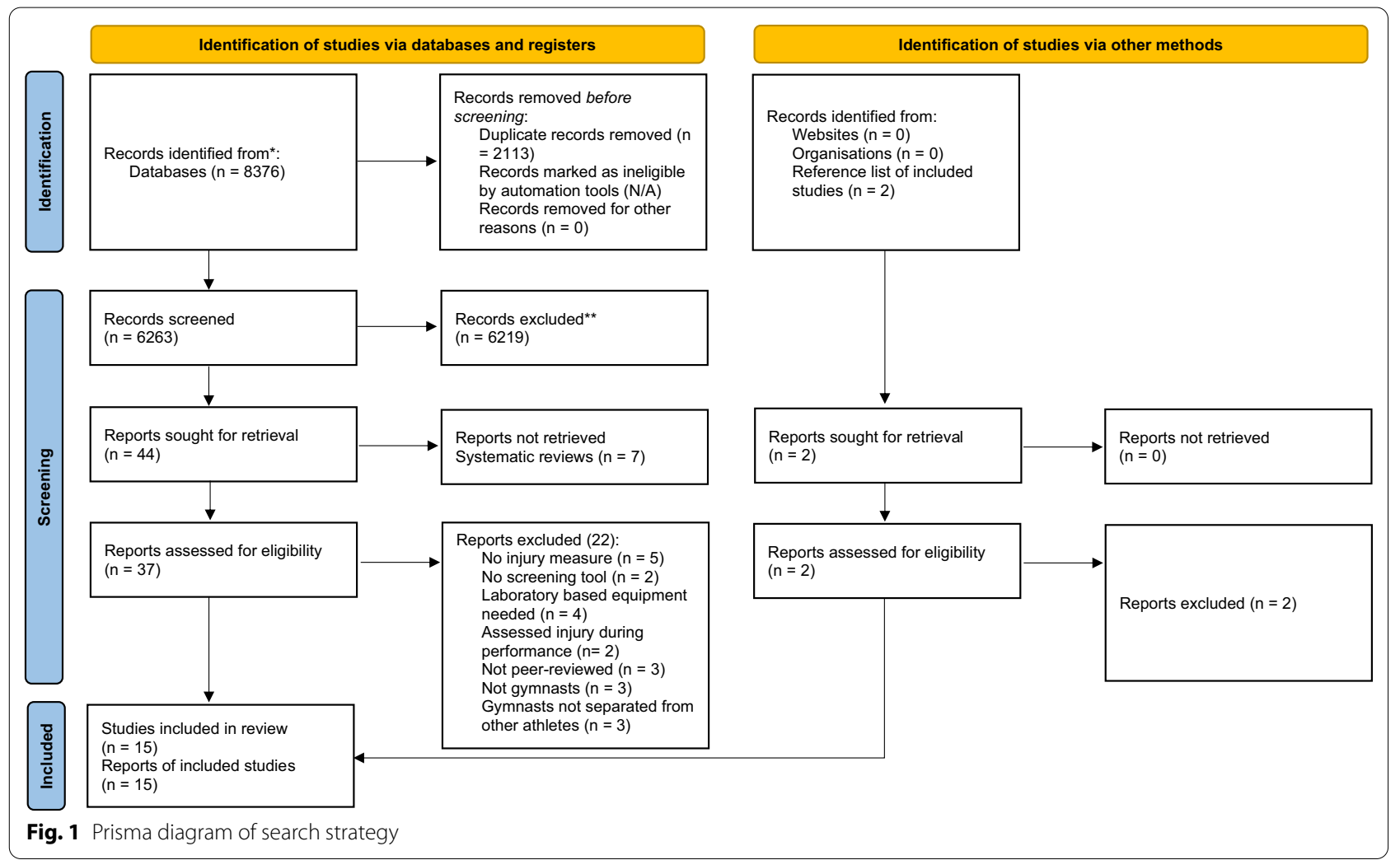

from a precompetitive level [25], 3 studies used competitive-level gymnasts [4, 26, 28], 2 studies used a mixed group of gymnasts $[2,27]$ and 1 study included gymnasts attending a course and the level was unclear [24].

\section{Age and Sex}

Four studies used gymnasts under 18 years old [4, 25, $26,28]$ and 2 studies included gymnasts above 18 years old [1, 24]. Four studies included gymnasts across the age range; 6-18 years [2, 9, 21, 22], 1 study 13-19 years [23], 1 study 10-21 years [27], 1 study $8-18$ years [29] and 2 studies $16-28$ years $[30,31]$. Seven studies included females only $[1,4,9,23,24,26,27], 6$ studies were mixed $[2,21,22,25,28,29]$ and 2 studies included males only $[30,31]$.

\section{Injury Definition and Diagnosis}

Eight studies provided a definition of injury [1, 4, 21, 22, 24, 29-31] and 7 studies did not define injury [2, 9, 23, 25-28]. Four studies investigated/defined pain [9, 23, 25, 28]. In 5 studies [22, 24, 29-31] injury was self-reported, and in 4 studies pain was self-reported $[9,23,25,28]$. In 3 studies [2, 4, 27] the method of diagnosis was unclear. In 1 study certified trainers recorded injury data [21] and in 1 study the "researchers" assessed the injury [26]. In one study, the diagnosis was provided by a "certified athletic trainer, physical therapist, physician or chiropractor" [1]. Seven studies investigated a specific type of injury/pain: low back pain $[9,23,28]$, wrist pain [25], ankle injury [22, $30]$ and wrist injury [31].

\section{Statistical Analysis}

Seven studies used regression models or risk measurement $[1,2,4,9,25,27,28]$, and 8 studies used inferential analysis that did not include regression or risk measurements [21-24, 26, 29-31]. Six studies used both types of statistical analysis $[2,4,9,25,27,28]$.

\section{Range of Motion}

Eight studies [4, 9, 22, 24, 26-29] investigated the relationship between range of motion (ROM) and injury. Only the screening data for significant results are reported here.

Kirby et al. [26] investigated 60 female gymnasts and 35 aged matched non-athletic controls for musculoskeletal symptoms and flexibility ROM and reported that those gymnasts who had greater ROM on toe touching also had greater low back pain $(P=0.043)$ when compared with the controls. Lindner and Caine [4] reported that injured gymnasts had better sit and reach $(P<0.05)$ and higher leg raise forward left $(P<0.02)$ results than uninjured gymnasts but were not different in the remaining 


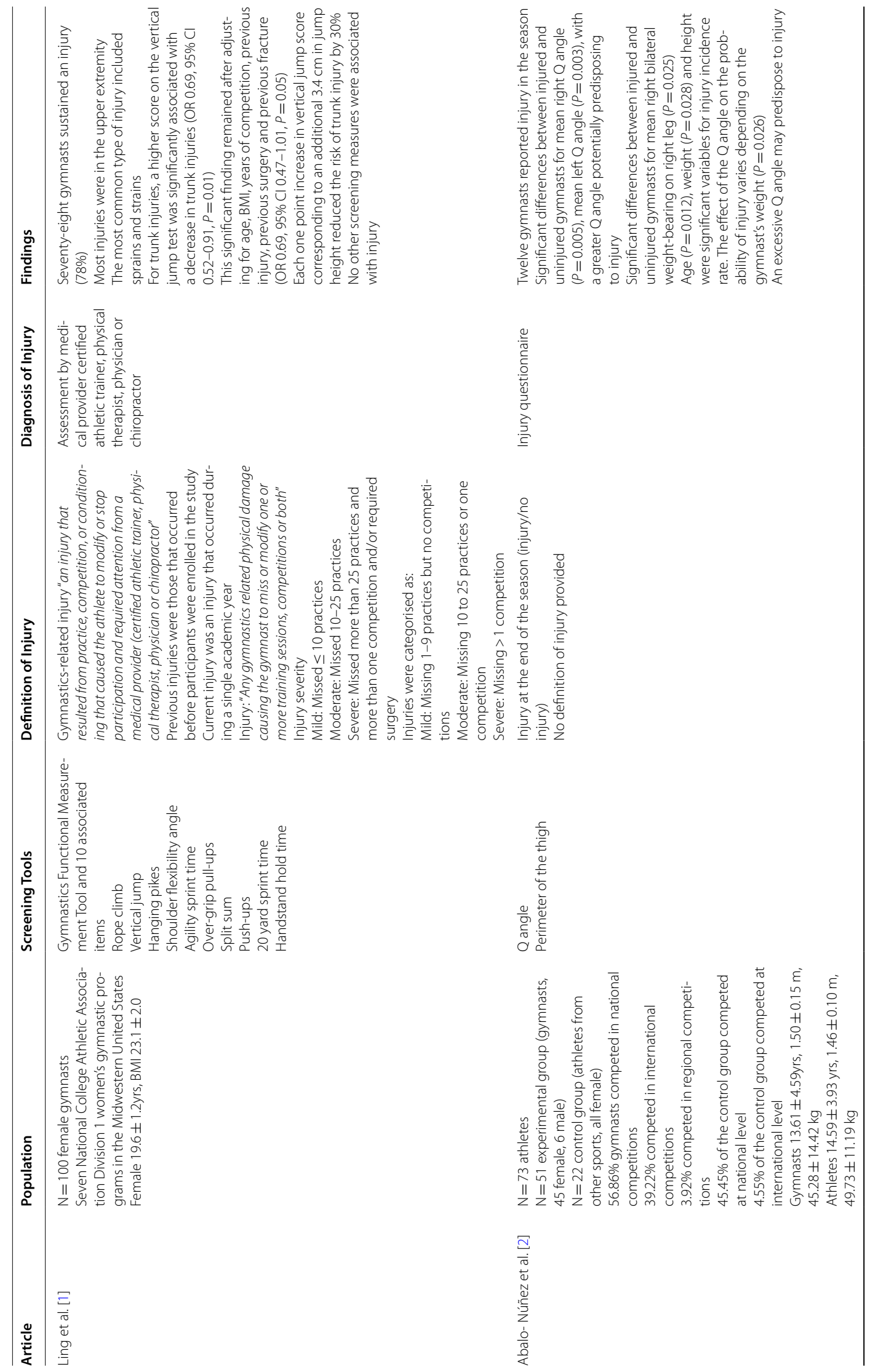




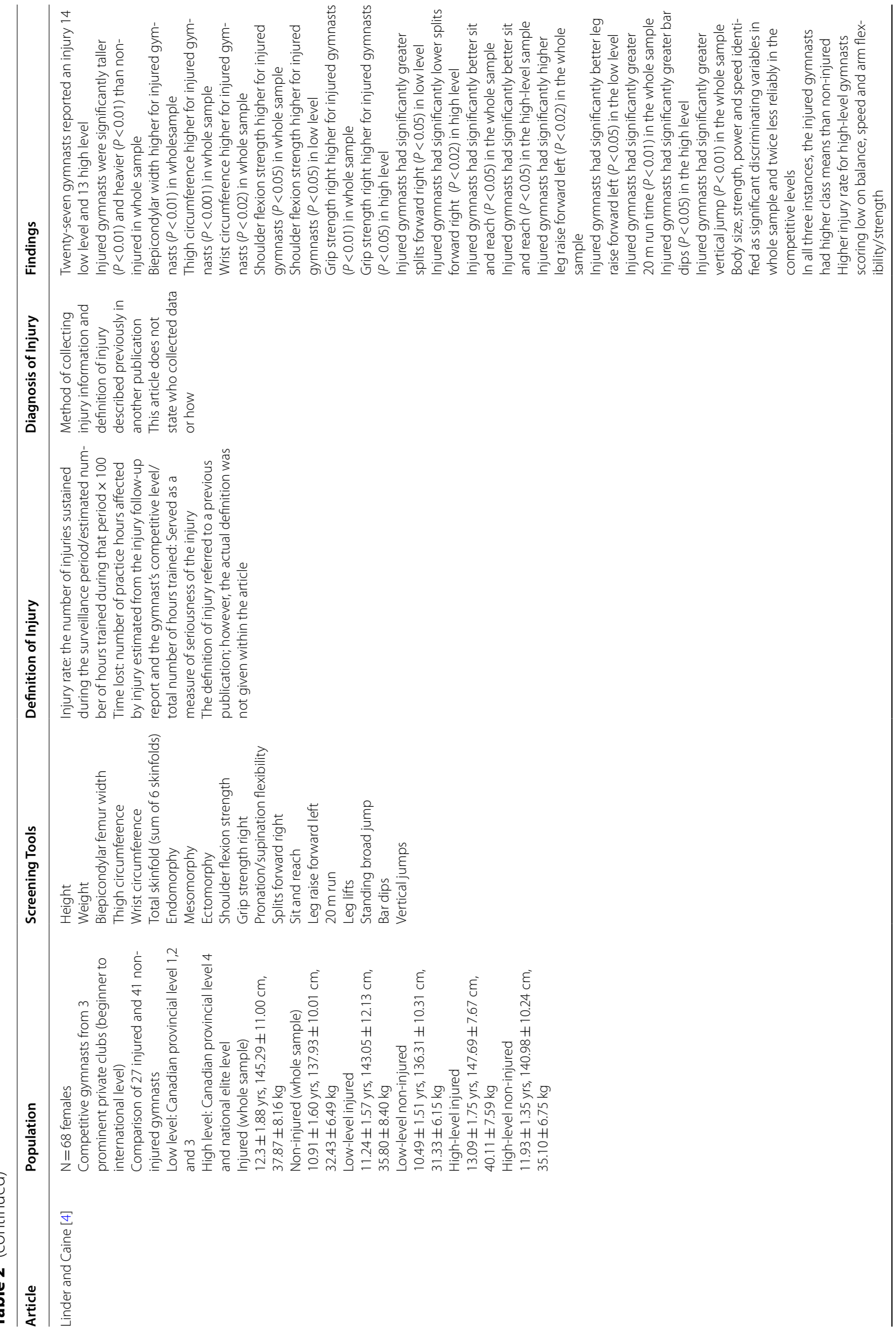




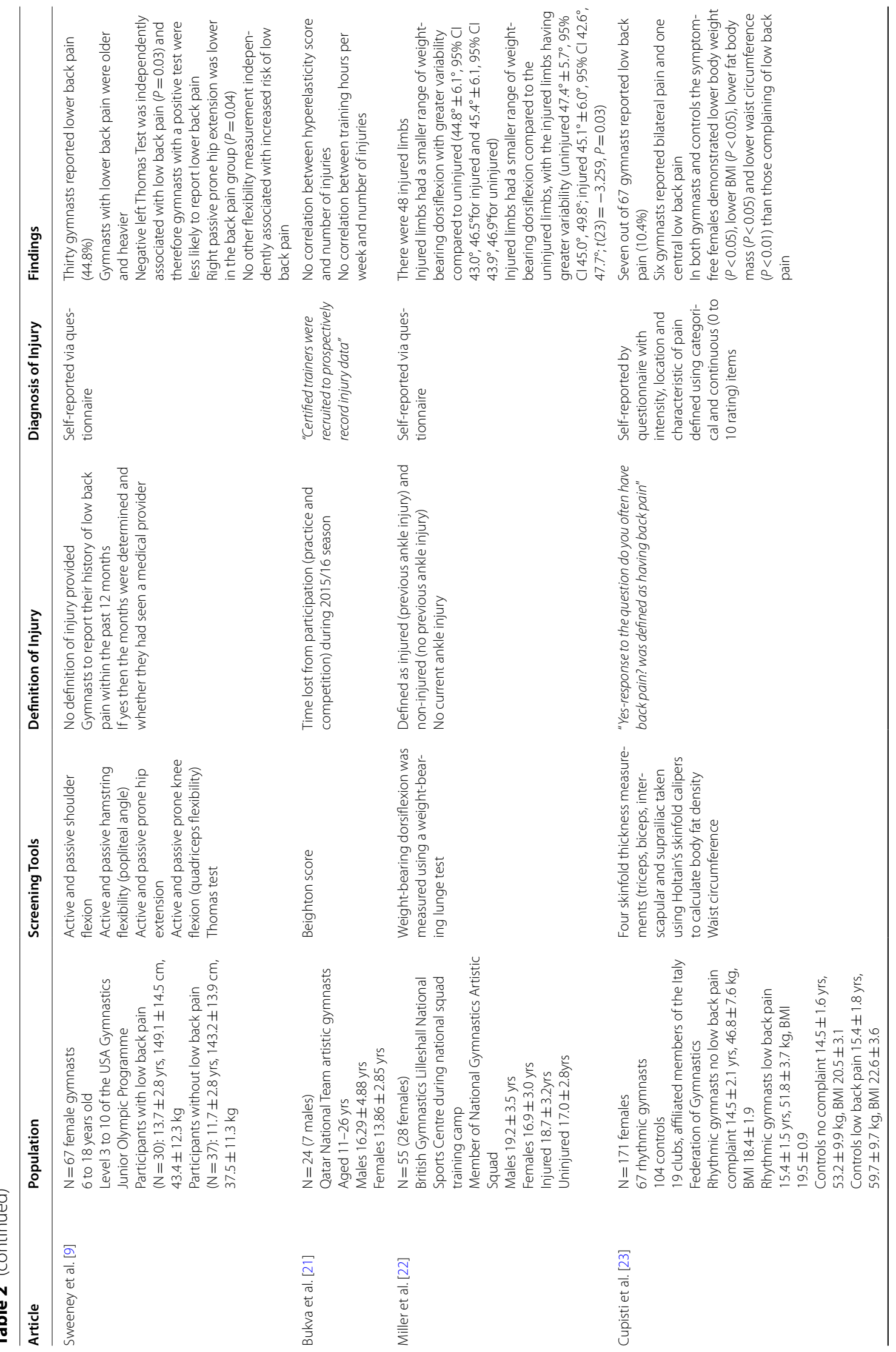




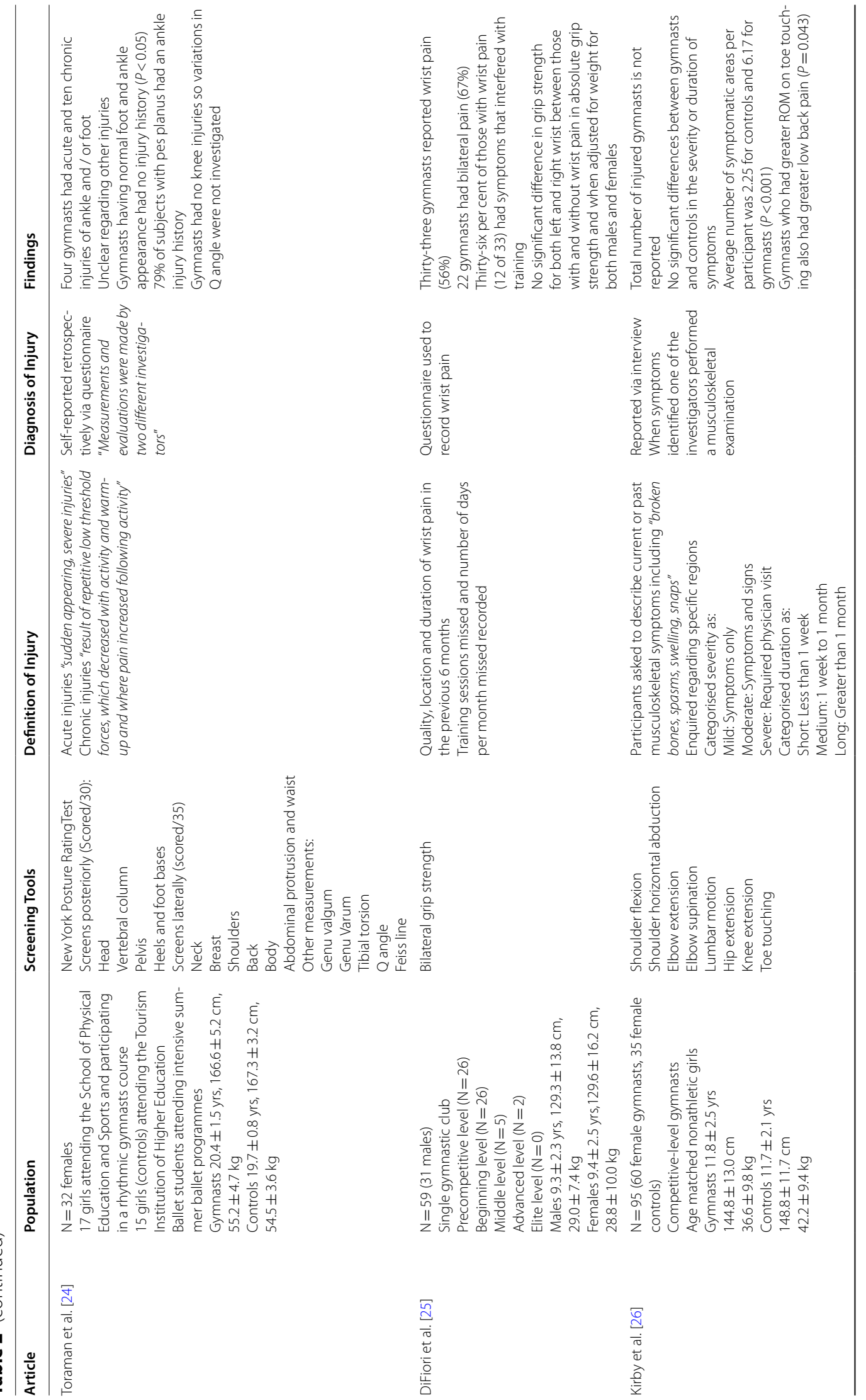




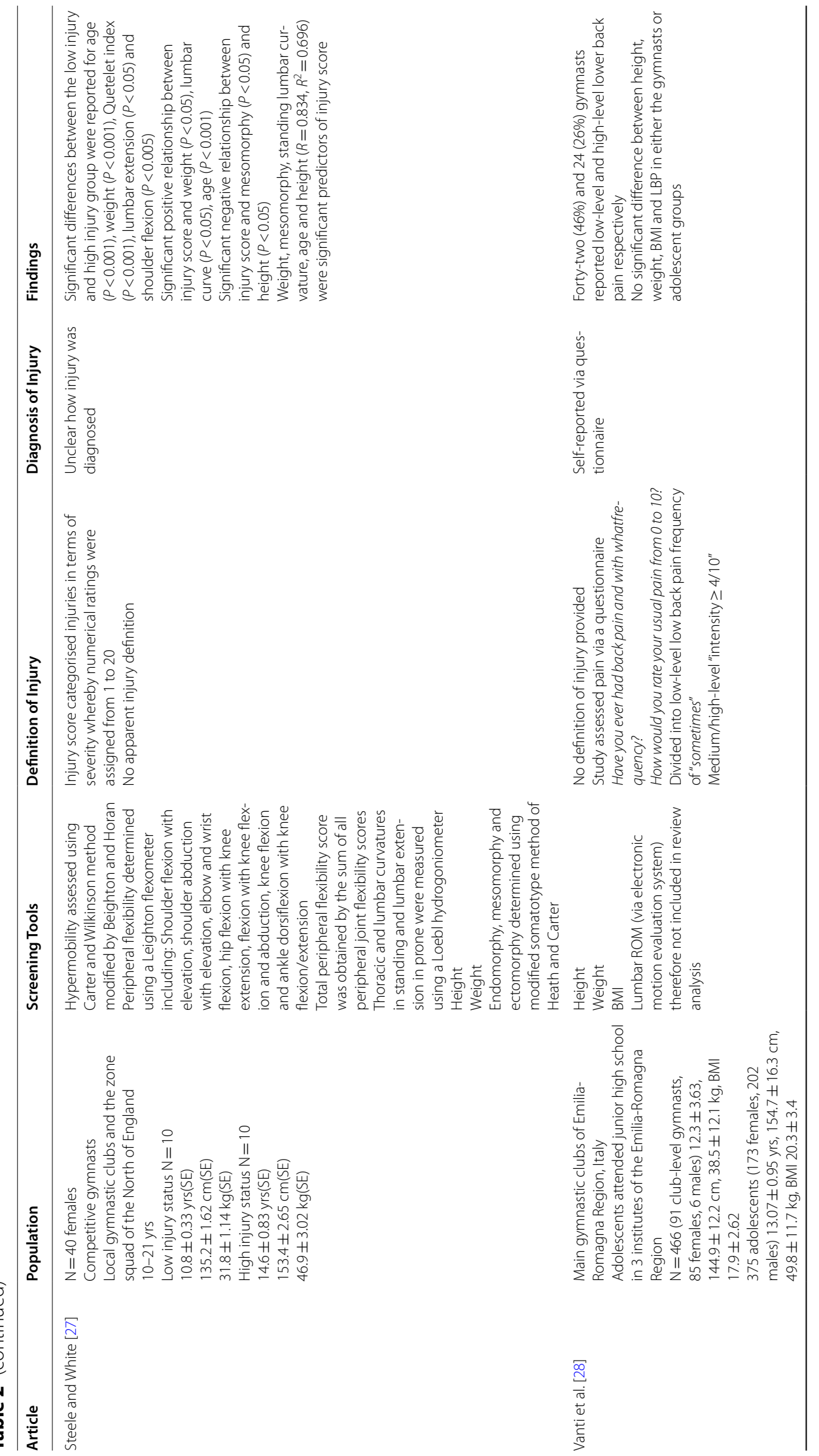




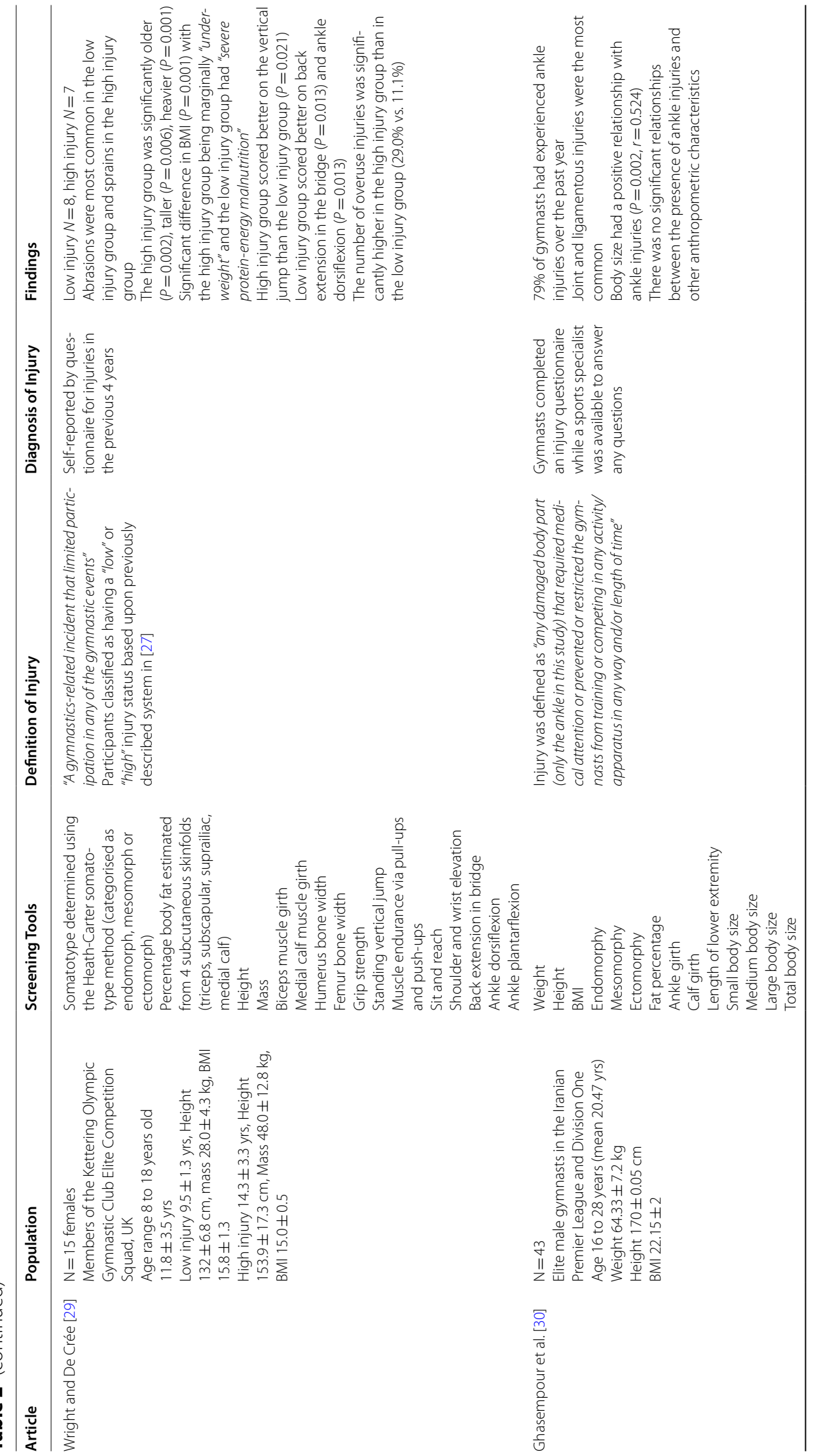




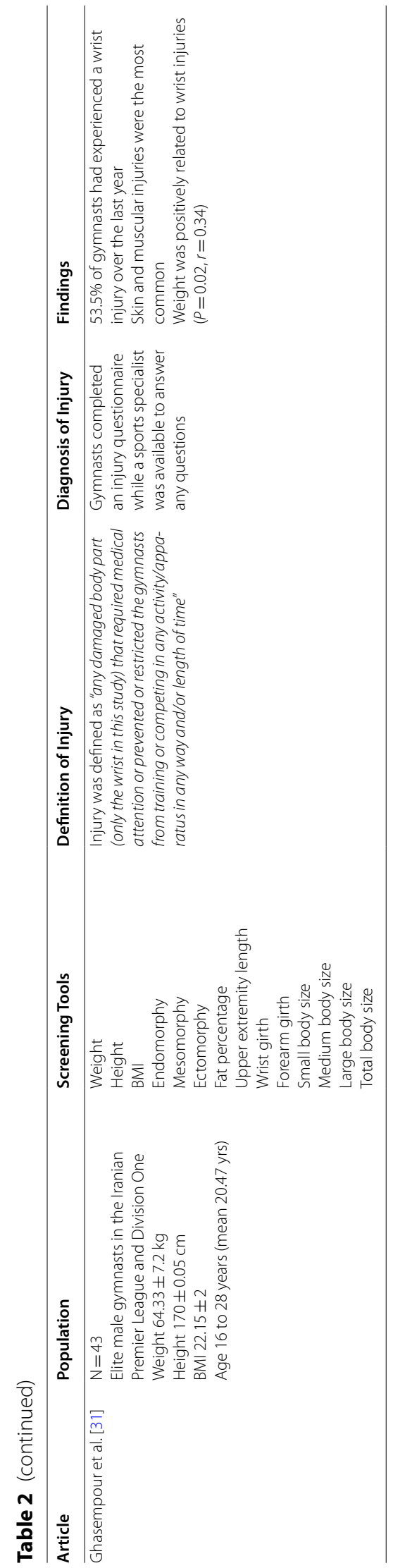


flexibility tests. When gymnasts were separated into high- and low-level performance groups, injured gymnasts in both groups had significantly greater forward splits on the right side (where the right leg is extended forward and the left leg positioned to the rear of the trunk) compared with uninjured gymnasts (high level $P<0.02$, low level $P<0.05)$. In the low-level group only, injured gymnasts had significantly higher leg raise forward on the left side $(P<0.05)$. Injured high-level gymnasts had better sit and reach scores than high-level uninjured gymnasts $(P<0.05)$. Finally, high-level injured gymnasts who scored lower on a combination of balance, speed and arm flexibility/strength variables had higher injury rates $(P=0.0495)$ in the stepwise regression model.

Miller et al. [22] reported that injured limbs had a smaller range of weight-bearing ankle dorsiflexion with greater variability compared to uninjured $\left(44.8^{\circ} \pm 6.1^{\circ}\right.$, $95 \%$ confidence interval (CI) $43.0^{\circ}-46.5^{\circ}$ for injured and $45.4^{\circ} \pm 6.1^{\circ}, 95 \%$ CI $43.9^{\circ}-46.9^{\circ}$ for uninjured) in 55 national-level artistic gymnasts. Wright and De Crée [29] reported that in 15 gymnasts the lower injury group scored lower on ankle dorsiflexion $(62.3 \mathrm{~cm} \pm 8.0$ vs. $75.9 \mathrm{~cm} \pm 10.3, P=0.013)$ and back extension in the bridge $(22.6 \mathrm{~cm} \pm 1.9$ vs. $25.5 \mathrm{~cm} \pm 4.9, P=0.013)$ which they stated as better performances when compared with the high injury group. Steele and White [27] reported low injury groups had superior scores for lumbar extension; $46.7^{\circ} \pm 2.94$ (mean \pm standard error (SE)) versus $59.8^{\circ} \pm 4.32($ mean $\pm \mathrm{SE})(P<0.05)$ and shoulder flexion $253.8^{\circ} \pm 3.13(\mathrm{SE})$ versus $240.1^{\circ} \pm 4.91(\mathrm{SE})(P<0.05)$ when compared to high injury groups, respectively, in 40 North of England squad female gymnasts.

\section{Anthropometrics and Posture}

Nine studies investigated the relationship between anthropometric values and/or posture and injury [2, 4, $23,24,27-31]$. Only the screening data for the significant results are reported here.

In a study investigating low back pain in 67 female rhythmic gymnasts and 104 controls, skinfold measurements were obtained at the triceps, biceps, inter-scapular and suprailiac to calculate body density [23]. In both gymnasts and controls the symptom-free females demonstrated lower body weight $(P<0.05)$, lower body mass index (BMI), $P<0.05)$, lower fat body mass $(P<0.05)$ and lower waist circumference $(P<0.01)$ than those with lower back pain [23]. Lindner and Caine [4] reported that in 68 competitive gymnasts, injured gymnasts were significantly taller $(P<0.01)$ and heavier $(P<0.01)$ than non-injured. Injured gymnasts had significantly greater biepicondylar femur width $(P<0.01)$, thigh circumference $(P<0.001)$, wrist circumference $(P<0.02)$ in the whole sample $(P<0.01)$ and a significantly greater biepicondylar femur $(P<0.05)$ and thigh circumference $(P<0.01)$ in high-level gymnasts. Thigh circumference $(P<0.01)$ and wrist circumference $(P<0.02)$ were significantly greater in injured gymnasts in the low-level sample.

Abalo-Núñez et al. [2] reported that in 51 nationaland international-level gymnasts, age $(P<0.01)$, weight $(P<0.01)$ and height $(P<0.01)$ were significant variables for injury incidence rate. In 40 female competitive gymnasts, height, weight and somatotype were determined and significant differences existed between low injury status and high injury status groups for age $(P<0.001)$, weight $(P<0.001)$ and BMI $(P<0.001)$ [27]. There was a significant positive relationship between injury score and weight $(P<0.05)$ and age $(P<0.001)$ and a significant negative relationship between injury score and mesomorphy $(P<0.05)$ and height $(P<0.05)$. Postural measurements of thoracic and lumbar curvatures and lumbar extension were taken using a Loebl hydrogoniometer and a significant difference existed between the low injury and high injury group for lumbar extension $(P<0.05)$, and there was a significant positive relationship between injury score and lumbar curve $(P<0.05)$. Unfortunately, it was unclear how body size was measured in this paper. Abalo-Núñez et al. [2] also reported a significant difference between injured and uninjured gymnasts for mean right $Q$ angle $(P=0.005)$, mean left $Q$ angle $(P=0.003)$ and the effect of $Q$ angle on the probability of injury varied depending upon the gymnast's weight $(P=0.026)$.

Toraman et al. [24] investigated posture using the New York Posture Rating Test which screens various parts of the body in 17 female gymnasts and a sedentary female control group attending a rhythmic gymnastics course and reported that gymnasts with normal foot and ankle appearance had no injury history $(P<0.05)$ while $79 \%$ of "subjects" with pes planus had an ankle injury history. However, the number of gymnasts within this percentage was not reported. Wright and DeCrée [29] determined somatotype and BMI utilising a 4-skinfold method at the triceps, subscapular, suprailiac and medial calf in 15 international-level gymnasts and classified injury status as low or high. The high injury group was significantly older $(P=0.002)$, taller $(P=0.006)$, heavier $(P=0.001)$ and there was a significant difference in BMI $(P=0.001)$ with the high injury group marginally "underweight" and the low injury group demonstrating "severe protein energy malnutrition"; however, the authors did not define these terms.

Ghasempour et al. [30] investigated ankle injuries in 60 elite male gymnasts of which injury data were recorded for 40 gymnasts. They reported that body size had a significant positive relationship with ankle injuries $(P=0.02$, $r=0.524)$. Ghasempour et al. [31] investigated wrist injuries in 43 elite male gymnasts and reported that body 
weight had a significant positive relationship $(P=0.02$, $r=0.34)$ and that heavier gymnasts suffered more injuries.

\section{Hypermobility}

Two studies reported no significant relationship between hypermobility scores, measured using the Beighton modification of the Carter-Wilkinson criteria (cut-off $\geq 5$ ), and injury $[21,27]$.

\section{Clinical Diagnostic Tests}

One study [9] investigated the relationship between clinical diagnostic tests and injury but only used one clinical diagnostic test, namely the Thomas test alongside a series of ROM measurements. Only the screening data for significant results are reported here.

A negative left Thomas test was independently associated with low back pain $(P=0.03)$, and therefore, gymnasts with a positive test were less likely to report low back pain.

\section{Movement Screening Tools}

One study investigated the relationship between movement screening tools and injury [1]. This study used the Gymnastics Functional Measurement Tool which consists of 10 items and investigated 100 female gymnasts. Only the screening data for significant results are reported here.

For trunk injuries a higher score on the vertical jump test was significantly associated with a decrease in trunk injuries (OR 0.69, 95\% CI 0.52-0.91, $P=0.01$ ) and for each one point increase in vertical jump score which corresponded to an additional $3.4 \mathrm{~cm}$ the risk of trunk injury was reduced by $30 \%$.

\section{Muscle Control, Strength, Power and Endurance}

Three studies investigated muscle control, strength, power and endurance $[4,25,29]$. Only the screening data for significant results are reported here.

DiFiori [25] investigated the relationship between bilateral grip strength and wrist pain in 59 precompetitivelevel gymnasts and reported no significant difference between those with and without wrist pain in relation to absolute grip strength and when adjusted for weight for both males and females. Lindner and Caine [4] compared 27 injured and 41 non-injured gymnasts and reported shoulder flexion strength was greater in injured gymnasts for the whole sample $(P<0.05)$ and low-level sample $(P<0.05)$. Grip strength right for the whole sample $(P<0.01)$ and high-level sample $(P<0.05)$ was greater in injured gymnasts compared to uninjured gymnasts. Injured gymnasts had significantly greater bar dips in the high-level gymnasts $(P<0.05)$ and significantly greater vertical jump $(P<0.001)$ in the whole sample. In addition, strength, power and speed were identified as significant discriminating variables with injured gymnasts having higher class means than non-injured. Wright and De Crée [29] reported that the "high injury status" group had a better vertical jump than the "low injury status" group $(P=0.021)$.

\section{Other Screening Measurements}

One study investigated $20 \mathrm{~m}$ run time [4] and injured gymnasts had significantly faster times in the whole sample $(P<0.01)$ compared with uninjured gymnasts. All other findings were reported at a non-significant level.

\section{Discussion}

To the best of our knowledge, this is the first systematic literature review to investigate which screening tools can predict injury in all genres, levels and ages of gymnasts.

\section{Methodological Quality}

The mean score using the methodological quality tool was 13.1 points (range $10-17$ points) with all studies being of level 4 evidence. All studies provided a description of the screening tools used. As with many injury studies, the literature is limited by the varying definitions of musculoskeletal injury and by who defined the injury. Eight studies provided a definition of injury [1, $4,21,22,24,29-31]$ and 7 studies did not define injury $[2,9,23,25-28]$. Seven studies investigated a specific type of injury/pain: low back pain $[9,23,28]$, wrist pain [25], ankle injury [22, 30], wrist injury [31]. Four studies investigated/defined pain [9, 23, 25, 28]. In 5 studies [22, 24, 29-31] injury was self-reported, and in 4 studies pain was self-reported $[9,23,25,28]$. In 3 studies [2, 4, 27] the method of diagnosis was unclear. In 1 study certified trainers recorded injury data [21] and in 1 study the "researchers" assessed the injury [26]. In one study, the diagnosis was provided by a "certified athletic trainer, physical therapist, physician or chiropractor" [1]. As a minimum, it is recommended that studies should provide a definition of musculoskeletal injury and have the diagnosis made by a medical professional ideally a physical therapist/physiotherapist or doctor as self-reporting by athletes has a greater potential for misdiagnosis [18].

The reporting of the reliability of the screening tools used is important and was reported in 2 studies [1,22] but no researchers reported the reliability within their own study. Data comparison was limited by some studies categorising gymnastic data into low-level and high-level gymnasts [4] and low injury and high injury status [27, 29]. Four studies that investigated pain $[9,23,25,28]$ were included to improve the depth of the review due to the 
terms often being used interchangeably; however, pain is different to injury and therefore any comparisons are required to consider the subjective nature of pain which is not always indicative or synonymous with injury. The development of chronic pain can be due to factors other than the cause of pain [33] and stress and environmental factors may require consideration. Kirby et al. [26] investigated symptoms and this requires consideration when interpreting the literature. Future research should clearly differentiate between pain and injury.

\section{Range of Motion}

Five studies reported significant findings between ROM and injury; however, these findings were across a number of locations and comparison included different/missing musculoskeletal injury definitions, measurements taken, mix of genres, levels and ages of gymnasts.

Two studies demonstrated significant findings for ankle dorsiflexion [22, 29]. Injured limbs had a smaller range of weight-bearing dorsiflexion with greater variability compared to uninjured in artistic gymnasts [22]. However, comparison with [29] is limited as [22] only investigated ankle injuries and had a varying methodology dependent upon the objective. For objective 1, data were used for all participants and each limb was considered individually while objective 3 only used participants with a history of a unilateral previous injury. Gymnasts in the lower injury group scored better on ankle dorsiflexion and back extension in the bridge than the high injury group [29]. It was suggested that less flexible individuals might be more likely to sustain injuries.

Steele and White [27] used the same injury classification system as [29] and the finding of increased back/ lumbar extension in high injury groups is in agreement. However, with these findings it is not clear if extension was measured using the same protocol. Shoulder flexion was lower in the high injury group; however, no explanation was provided for this finding [27]. The finding of Kirby et al. [26] that those gymnasts who had greater ROM on toe touching also had more low back pain may relate to shoulder flexion and back ROM as both regions contribute to this movement. However, as this was a "pain" study the complex relationship between pain and injury and different methodology restricts comparison. The sit and reach test involves back and arm ROM and [4] reported that injured gymnasts had significantly better sit and reach in the whole sample and in the high-level sample. High-level gymnasts scored low on arm flexibility and had higher injury rates. The suggestion was made that optimal levels of flexibility may exist for gymnastics with too much or too little flexibility increasing the injury risk; however, specific values were not suggested.
Due to the inconsistency of the results, it is unclear if ROM is a significant predictor of injury in gymnasts.

\section{Anthropometrics and Posture}

Seven studies reported significant findings between anthropometric data, posture and injury $[2,4,23,24$, 29-31]; however, these findings were across a number of locations and comparison included different/missing musculoskeletal injury definitions, measurements taken, mix of genres, levels and ages of gymnasts.

In rhythmic gymnasts and controls the symptom-free females demonstrated lower body weight, lower BMI, lower fat body mass and lower waist circumference than those with lower back pain [23]. Wright and De Crée [29] classified injury status as low or high and reported that high injury group was taller, heavier and for BMI the high injury group was marginally "underweight" and the low injury group demonstrated "severe protein energy malnutrition." Abalo-Núñez et al. [2] reported that weight and height were significant variables for injury incidence rate and that abnormal alignments that result in unequal weight distribution may influence lower limb injury development. In female competitive gymnasts there was a significant positive relationship between injury score and weight and a significant negative relationship between injury score, mesomorphy and height [27]. It was concluded that females with poor musculature, short stature who were relatively heavy were more injury prone. Postural measurements of thoracic and lumbar curvatures and lumbar extension demonstrated a significant difference between the low injury and high injury group for lumbar extension, and there was a significant positive relationship between injury score and lumbar curve with the suggestion that hyperlordosis may predispose towards back injury [27].

Lindner and Caine [4] reported that injured gymnasts were significantly taller and heavier than non-injured in agreement with [29]. Injured gymnasts had significantly greater biepicondylar femur width, thigh circumference, wrist circumference in the whole sample and a significantly greater biepicondylar femur and thigh circumference in injured high-level compared to noninjured high-level gymnasts. Thigh circumference and wrist circumference were significantly greater in injured gymnasts than non-injured in the low-level sample. Two studies using the same participants [30,31] highlighted that greater body size was related to ankle injuries and that greater body weight was related to wrist injuries. With ankle injuries it was suggested that increases in height and weight increase the degree of inversion torque that the ankle complex must withstand [30]. Within this study $76 \%$ of ankle injuries occurred in the landing phase and the high volume of landings associated with 
gymnastics increase the injury risk. For wrist injuries, heavier gymnasts are more susceptible to wrist injuries due to increased loading and because gymnastics is a sport that requires the wrist to be a wrist bearing joint [31]. However, this study did not record training or competition time or the type of apparatus used which could influence the results.

One study reported that the Q angle [2] was related to injury and it was concluded that an excessive $\mathrm{Q}$ angle could predispose to injury particularly the left $\mathrm{Q}$ angle, however, what measurement was considered an excessive $Q$ angle was not stated. Interestingly the effect of the $Q$ angle on injury was related to the gymnast's weight. It was concluded that an increased Q angle may create a lateral valgus force vector that results in a misalignment of force transmission, and abnormal lateral movements and an increased risk of injury.

The interpretation of results was restricted by the different methodologies utilised which resulted in a variety of measurements been recorded. Lindner and Caine [4] and Steele and White [27] reported height, weight and body type (ectomorphy, mesomorphy, endomorphy) with no calculation of BMI. Abalo-Núñez [2] only presented height and weight with no separation of male and female data. Cupisti et al. [23] reported weight, BMI, fat body mass and waist circumference. Ghasempour et al. [30, 31] measured height, weight, BMI, fat percentage, body type (ectomorphy, mesomorphy, endomorphy) and body size and although Wright and De Crée [29] used similar variables with the exception of body size, comparison of results is limited by the age, gender and injury type differences. Therefore, based upon these methodological differences it is not possible to present specific values associated with injuries that could be used for injury prevention guidelines. However, from the limited number of studies available it is possible that taller and heavier gymnasts might be more susceptible to injury, however, what values define a tall and heavy gymnast is unclear.

\section{Hypermobility}

Despite recognised screening tools such as the Beighton score existing, only two studies [21,27] have investigated the relationship between hypermobility and gymnastic injury. Neither study reported any significant findings, and currently it appears hypermobility is not a significant predictor of injury in gymnastics.

\section{Clinical Diagnostic Tests}

Only one study [9] investigated the relationship between clinical diagnostic tests (Thomas test) and reported that a negative left Thomas test was independently associated with low back pain and therefore gymnasts with a positive test were less likely to report low back pain. Potential explanations for this finding were the asymmetrical nature of gymnastics in relation to having a dominant limb potentially leading to tightness on one side, however, as leg dominance was not recorded it was acknowledged that this theory was speculative. Therefore, the evidence base is currently limited to one pain-based lower back study and requires further research.

\section{Movement Screening Tools}

One study investigated the relationship between movement screening tools and injury [1] and reported that with the Gymnastics Functional Measurement Tool trunk injuries were reduced with a higher vertical jump score with an additional $3.4 \mathrm{~cm}$ reducing the risk of trunk injury by $30 \%$. It was hypothesised that inadequate hip extensor strength might lead to a lower vertical jump height and increase vulnerability to trunk injuries; however, hip extensor strength was not measured directly. This gymnastic specific screening tool would benefit from further research to investigate the relationship with injury development further. With only one movement screening tool used this is an area for further research.

\section{Muscle Control, Strength, Power and Endurance}

Two studies reported a relationship between muscle control, strength, power and endurance and injury $[4,29]$. Lindner and Caine [4] compared injured and non-injured gymnasts and reported shoulder flexion strength (whole sample), shoulder flexion strength (low level) and grip strength right (whole sample), grip strength right (high level) were greater in injured gymnasts; however, no explanation was provided for this finding. Injured gymnasts had significantly greater bar dips in the (high level) gymnasts and significantly greater vertical jump (whole sample). It was suggested that bigger and stronger gymnasts were also older and therefore more likely to perform risky skills and practice longer although neither variable was analysed. In addition, strength, power and speed were identified as significant discriminating variables with injured gymnasts having higher class means than non-injured. This relationship between vertical jump and injury was also reported by [29] who demonstrated that the "high injury group" had a better vertical jump than the "low injury" group but is in contrast to the findings of [9].

Due to the inconsistency of results, it is unclear if muscle control, strength and power are a significant predictor of injury in gymnasts.

\section{Other Screening Measurements}

Findings were limited to one study [4], which reported that injured gymnasts had significantly greater $20 \mathrm{~m}$ run speed in the whole sample; however, no discussion of 
this finding was provided. These findings require further investigation.

\section{Limitations and Recommendations for Future Research}

No measurements were eligible for further analysis via a meta-analysis and the identification of which musculoskeletal screening tools may predict injury proved difficult due to the lack of standardisation of methods and data reporting. Our aim was to perform a meta-analysis of several measurements; however, this was prevented by poor clarity of methodology and variation in the measurement of parameters. Furthermore, the included literature was limited by small sample size, contrasting injury surveillance reporting and risk factor identification and failure to consider confounding variables. Some studies focussed on the identification of one specific injury type, and this should be considered when evaluating the evidence. Gymnasts often continue to compete when injured and this is particularly relevant at elite level. Gymnasts may have pain, but not necessarily be injured and this should be considered when reviewing injury and pain studies. Gymnastic performance requires sufficient energy availability to maintain health and reduce injury risk, and this is particularly important in female rhythmic gymnastics [34]. Therefore, it is recommended that future studies consider reporting nutrition intake to allow consideration of energy availability. The systematic review was limited to English language studies, and potentially, some studies may not have been included.

This study has provided information regarding the different genres, level and ages of gymnasts as all may influence study findings. Incomplete description of inclusion/ exclusion criteria (9 studies) and reporting of dropouts was present ( 5 studies) which can restrict interpretation. Screening tool reliability requires greater consideration as an unreliable tool may result in a lack of measurement consistency and studies should consider inter and intrarater reliability and validity. Studies should record training and competition duration to allow determination of injury rate and exposure data. Studies should report the injury severity and duration and provide an injury definition. It is also important that studies report who provides the diagnosis of injury to allow appropriate evaluation of potential clinical knowledge.

Prospective injury cohort studies are preferential in comparison with retrospective studies, and power calculations are advocated to determine sample size. However, only 4 studies were prospective. Future research should consider multivariate regression models if the aim is to determine the predictors of injury and if considering multiple risk factors should control for confounding variables and consider the potential interaction of those measures that are screened. One study [1] provided the following: (1) prospective design, (2) an injury definition, (3) a diagnosis by a physical therapist/physiotherapist or doctor and (4) the use of regression models or risk measurement. These factors represent good practice in investigating screening tools as a predictor of injury.

\section{Conclusions}

This systematic review is the first to collate and critically appraise musculoskeletal screening tools as a predictor of gymnastic injury and uses an effective scoring tool that recognises the importance of key factors including injury reporting and reliability. Some evidence existed for measurement of height and mass within the systematic review as taller and heavier gymnasts might be more susceptible to injury. Only one study has utilised a movement screening tool, namely the Gymnastics Functional Measurement Tool. Future studies that investigate the ability of screening tools to predict injury should be prospective, use predictive statistics, report the reliability of the tests and consider confounders. A specific definition of injury should be provided and diagnosis provided by an appropriate medical professional.

\section{Abbreviations \\ BMI: Body mass index; Cl: Confidence interval; OR: Odds ratio; ROM: Range of motion; RR: Relative risk; SE: Standard error; Q angle: Quadriceps angle.}

\section{Acknowledgements \\ Not applicable.}

\section{Declarations}

\section{Authors' Information}

Dr Ross Armstrong is a Chartered Physiotherapist with 20 years of experience specialising in musculoskeletal physiotherapy. His PhD was titled: Musculoskeletal Screening: Implications for Injury and Performance. He is a Senior Lecturer in Primary and Urgent Care and holds an MSc Sports and Exercise Medicine and has published within the domain of gymnastics and Exercise Medicine. He is a Cochrane-trained review author. Dr Nicola Relph is a specialist in biomechanics and Senior Lecturer in Epidemiology and has published systematic reviews and meta-analysis previously. She is a Cochrane-trained review author

\section{Authors' Contributions}

RA produced the original idea and conducted the literature search. He also reviewed and scored the returned articles. He wrote the manuscript. NR reviewed and scored the returned articles. She assisted with the writing of the manuscript. Both authors read and approved the final manuscript.

\section{Funding}

The authors wish to thank the University of Cumbria Research Internal Fund which provided funding for the article processing cost.

\section{Availability of Data and Materials}

Data presented in this article are available in the associated studies, references are provided.

Ethics Approval and Consent to Participate

Ethical approval was not required for this systematic review and meta-analysis. 


\section{Consent for Publication}

Not applicable as this manuscript does not include any individual person's data.

\section{Competing Interests}

Ross Armstrong and Nicola Relph declare that they have no competing interests with the content of this article.

\section{Author details}

${ }^{1}$ Rehabilitation and Healthy Lives Research Group, Institute of Health, University of Cumbria, Carlisle, Cumbria CA1 2HH, England. ${ }^{2}$ Promoting Population Musculoskeletal Health Research Group, Faculty of Health and Social Care, Edge Hill University, Ormskirk, Lancashire L39 4QP, England.

Received: 20 May 2021 Accepted: 17 September 2021

Published online: 11 October 2021

\section{References}

1. Ling D, Sleeper M, Casey E. Identification of risk factors for injury in women's collegiate gymnastics with the gymnastics functional measurement tool. PM R. 2020;Jan;12(1):43-8. doi: https://doi.org/10.1002/pmrj. 12184. Epub 2019 Sep 12. PMID: 31063637.

2. Abalo-Núñez R, Gutiérrez-Sánchez A, Iglesias Pérez MC, Vernetta-Santana M. Injury prediction in aerobic gymnastics based on anthropometric variables. Sci Sports. 2018;33:228-36.

3. Schluz MR, Marshall SW, Yang J, Mueller FO, Weaver NL, Bowling M. A prospective cohort study of injury incidence and risk factors in North Carolina high school competitive cheerleaders. Am J Sports Med. 2004;32:396-405.

4. Lindner KJ, Caine DJ. Physical and performance characteristics of injured and injury free female gymnasts. J Hum Mov Stud. 1993;25:69-83.

5. Sands WA, Shultz BB, Newman AP. Women's gymnastics injuries. A 5-year study. Am J Sports Med. 1993;21:271-6.

6. Marshall SW, Covassin T, Dick R, Nassar LG, Agel J. Descriptive epidemiology of collegiate women's gymnastic injuries: national collegiate athletic association injury surveillance system, 1988-1989 through 2003-2004. J Athletic Train. 2007:42:234-40.

7. Meeusen R, Borms J. Gymnastic injuries. Sports Med. 1992;13:337-56.

8. Pettrone FA, Ricciardelli E. Gymnastic injuries: the Virginia experience. Am J Sports Med. 1987;15:59-62.

9. Sweeney EA, Daoud AK, Potter MN, Ritchie L, Howell DR. Association between flexibility and low back pain female adolescent gymnasts. Clin J Sports Med. 2019;29(5):379-83.

10. Edouard $P$, Steffen $K$, Junge A, et al. Gymnastics injury incidence during the 2008, 2012 and 2016 Olympic Games: analysis of prospectively collected surveillance data from 963 registered gymnasts during Olympic Games. Br J Sports Med. 2018;52:475-81.

11. Caine D, Cochrane B, Caine C, Zemper E. An epidemiologic investigation of injuries affecting young competitive female gymnasts. Am J Sports Med. 1989;17:811-20.

12. Van Mechelen W, Hlobil H, Kemper H. Incidence, severity, aetiology and prevention of sports injuries. Sports Med. 1992;14:82-9.

13. Gabbe BJ, Bennell KL, Wajswelner H, Finch CF. Reliability of common lower extremity musculoskeletal screening tests. Phys Ther Sports. 2004;5(2):90-7.

14. Chorba R, Chorba DJ, Bouillon LE, Overmyer CA, Landis JA. Use of a functional movement screening tool to determine injury risk in female collegiate athletes. N Am J Sports Phys Ther. 2010;5(2):47-54.
15. Armstrong R, Greig M. Injury identification: the efficacy of the functional movement screen in female and rugby union players. Int J Sports Phys Ther. 2018;13(4):605-17. https://doi.org/10.26603/ijspt20180605.

16. Armstrong R. Joint hypermobility in young gymnasts: implications for injury and performance. J Educ Health Sports. 2018;8(11):354-75. https:// doi.org/10.5281/zenodo.1493831.

17. PROSPERO (york.ac.uk)

18. Armstrong R, Relph N. Screening tools as a predictor of injury in dance. A systematic review and meta-analysis. Sports Med Open. 2018;4:33. https://doi.org/10.1186/s40798-018-0146-z.

19. Dallinga JM, Benjaminse A, Lemmink APM. Which screening tools can predict injury to the lower extremities in team sports? A systematic review. Sports Med. 2012;42(9):791-815.

20. Devillé WL, Buntinx F, Bouter LM, Montori VM, de Vet HCW, van der Windt DAWM, et al. Conducting systematic reviews of diagnostic studies: didactic guidelines. BMC Med Res Methodol. 2002;3:2-9.

21. Bukva B, Vrgoč G, Madić Dy, Sporiš G, Trajković N. Correlation between hypermobility score and injury rate in artistic gymnastics. J Sports Med Phys Fitness. 2019;59(2):330-4.

22. Miller $\mathrm{H}$, Fawcett $\mathrm{L}$, Rushton $\mathrm{A}$. Does gender and ankle injury history affect weightbearing dorsiflexion in elite artistic gymnasts? Phys Ther Sport. 2020;42:46-52.

23. Cupisti A, D’Alessandro C, Evangelisti I, Piazza M, Galetta F, Morelli E. Low back pain in competitive rhythmic gymnasts. J Sports Med Phys Fitness. 2004;44(1):49-53.

24. Toraman F, Yaman H, Erman A. The effect of postural deviations in the occurrence of sports injuries in gymnasts. Spor Helkimiliği Dergisi Clit. 1999;34:S179-88.

25. DiFiori JP, Puffer JC, Aish B, Dorey F. Wrist pain, distal radial physeal injury and ulnar variance in young gymnasts: does a relationship exist? Am J Sports Med. 2002;30(6):879-85.

26. Kirby RL, Simms FC, Symington VJ, Garner JB. Flexibility and musculoskeletal symptomatology in female gymnasts and age-matched controls. Am J Sports Med. 1981;9(3):160-4.

27. Steele VA, White JA. Injury prediction in female gymnasts. Br J Sports Med 1986;20(1):31-3.

28. Vanti C, Gasperini M, Morsillo F, Pillastrini P. Low back pain in adolescent gymnasts. Prevalence and risk factors. Scienza Riabiltativa. 2019;12(2):45-50.

29. Wright KJ, De Crée C. J Phys Ther Sci. 1998;10:87-92.

30. Ghasempour H, Rajabi R, Alizadeh MH, Tavania A. Ankle injuries of elite male Iranian gymnasts and anthropometric characteristics. Turk J Sport Exerc. 2013;15(3):35-41.

31. Ghasempour H, Rajabi R, Alizadeh MH, Nikro H. Correlation between elite male Iranian gymnast's wrist injuries and their anthropometric characteristics. Electron Phys. 2014;6(4):932-8.

32. Page MJ, McKenzie JE, Bossuyt PM, Boutron I, Hoffmann TC, Mulrow CD, et al. The PRISMA 2020 statement: an updated guideline for reporting systematic reviews. BMJ. 2021;372: n71. https://doi.org/10.1136/bmj.n71.

33. Loeser JD, Melzack R. Pain: an overview. The Lancet. 1999;353(9164):1607-9.

34. Silva MR, Paiva T. Poor precompetitive sleep habits, nutrients' deficiencies, inappropriate body composition and athletic performance in elite gymnasts. Eur J Sports Sci. 2015;6:726-35.

\section{Publisher's Note}

Springer Nature remains neutral with regard to jurisdictional claims in published maps and institutional affiliations. 